\title{
Channel Modelling for Free-Space Optical Inter-HAP Links Using Adaptive ARQ Transmission
}

\author{
S. Parthasarathy*a ${ }^{a}$, D. Giggenbach ${ }^{a}$, A. Kirstädter ${ }^{b}$ \\ ${ }^{\mathrm{a}}$ Institute of Communications and Navigation, German Aerospace Centre (DLR), D-82234 Wessling; ${ }^{\mathrm{b}}$ Institute of \\ Communication Networks and Computer Engineering, University of Stuttgart, D-70569 Stuttgart
}

\begin{abstract}
Free-space optical (FSO) communication systems have seen significant developments in recent years due to growing need for very high data rates and tap-proof communication. The operation of an FSO link is suited to diverse variety of applications such as satellites, High Altitude Platforms (HAPs), Unmanned Aerial Vehicles (UAVs), aircrafts, ground stations and other areas involving both civil and military situations. FSO communication systems face challenges due to different effects of the atmospheric channel. FSO channel primarily suffers from scintillation effects due to Index of Refraction Turbulence (IRT). In addition, acquisition and pointing becomes more difficult because of the high directivity of the transmitted beam: Miss-pointing of the transmitted beam and tracking errors at the receiver generate additional fading of the optical signal. High Altitude Platforms (HAPs) are quasi-stationary vehicles operating in the stratosphere. The slowly varying but precisely determined time-of-flight of the Inter-HAP channel adds to its characteristics. To propose a suitable ARQ scheme, proper theoretical understanding of the optical atmospheric propagation and modeling of a specific scenario FSO channel is required. In this paper, a bi-directional symmetrical Inter-HAP link has been selected and modeled. The Inter-HAP channel model is then investigated via simulations in terms of optical scintillation induced by IRT and in presence of pointing error. The performance characteristic of the model is then quantified in terms of fading statistics from which the Packet Error Probability (PEP) is calculated. Based on the PEP characteristics, we propose suitable ARQ schemes.
\end{abstract}

Keywords: FSO, Index of Refraction Turbulence, Scintillation, Pointing Error, Bit Error Probability, Packet Error Probability, Transmission Efficiency, Error Detection and Correction.

\section{INTRODUCTION}

Free-space optical (FSO) communication uses collimated laser beam to transmit and receive data. FSO link operates in diverse variety of applications such as satellites, High Altitude Platforms (HAPs), Unmanned Aerial Vehicles (UAVs), aircrafts, ground stations and other areas involving both civil and military situations. Applications that could benefit from FSO connections are those that have platforms with limited weight and space, require very high data links, and must operate in an environment where fiber-optic links are not practical such as between buildings across cities and space links [1]. FSO systems have significant advantages compared to conventional RF systems - overcoming interference, bandwidth exhaustion, providing higher data rates in multi-gigabit regime. FSO communication systems have its significant challenges due to different effects of the atmospheric channel. FSO channel primarily suffers from scintillation effects due to Index of Refraction Turbulence (IRT). In addition, acquisition and pointing becomes more difficult because of the high directivity of the transmitted beam: Miss-pointing of the transmitted beam and tracking errors at the receiver generate additional fading of the optical signal. The resulting power loss at the receiver is eventually weakening the overall performance of the communication system. HAPs are quasi-stationary vehicles like helium-filled airships that operate in the stratosphere well above civil air routes, jet-streams, and clouds, but substantially below orbiting satellites [2]. HAPs provide a platform for scientific, military, or commercial payloads at typical heights of 17 to $25 \mathrm{~km}$, where the impact of atmosphere on the FSO communication link is less severe than directly above ground. Figure 1 depicts FSO laser communication link scenarios between HAPs.

*swaminathan.parthasarathy@dlr.de; phone +49 8153 282681; www.dlr.delkn 


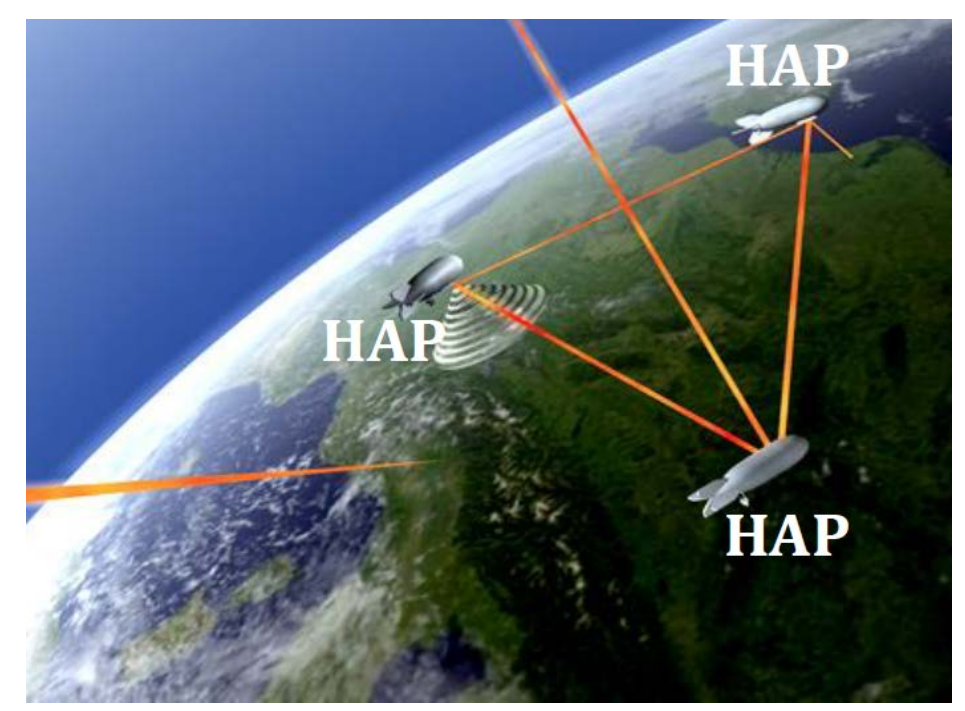

Figure 1.High Altitude Platform communication scenario [3].

Automatic Repeat reQuest (ARQ) schemes that are often used as Error Control technique for FSO communication systems with higher data rates to improve the impairments of the channel due to blockage or long fading events [4]. An ARQ scheme cannot be designed generic to all FSO applications. Rather, they have to be tailored for each specific atmospheric channel or scenario based on the fading statistics and the Bit Error Probability (BEP) characteristics of the FSO Inter-HAP channel. In this paper, therefore, we model a bi-directional symmetrical Inter-HAP FSO link. The InterHAP channel model is then investigated via simulations in terms of optical scintillation induced due to IRT and in presence of pointing error. The performance characteristic of the model is quantified in terms of Packet Error Probability (PEP) characteristics from which suitable ARQ schemes are proposed.

The remainder of the paper is organized as follows. The Inter-HAP link channel model is detailed in Section 2 . In Section 3, briefing of selected ARQ schemes, parameter definition and its performance characteristics is described. Performance results and comparison of ARQ schemes are given in Section 4. Finally, Section 5 discusses the results and concludes the paper.

\section{INTER-HAP CHANNEL MODELLING}

The optimum performance of a communication channel can be well characterized and efficiently implemented by modelling a channel with different parameters. In order to fully understand and characterize FSO communication between two HAPs it is important to model the channel based on different aspects [4].

\subsection{Atmospheric effects on Inter-HAP link}

\subsubsection{Optical Turbulence Profile}

Reliable values of physical stratospheric are required to model an Inter-HAP channel. Reliable and accurate measurement data of the turbulence are rare. For applications involving propagation along a horizontal path, it is customary to assume that the structure parameter $C_{n}^{2}$ remains essentially constant [1]. The altitude profile for the parameter $\mathrm{C}_{\mathrm{n}}{ }^{2}$ (which scales the atmosphere's IRT structure function) is generated using the Hufnagel-Valley (H-V) model [5]. The H-V model is described as a function of height as follows:

$$
C_{n}^{2}(h)=0.00594\left(\frac{w}{27}\right)^{2}\left(10^{-5} h\right)^{10} \exp \left(-\frac{h}{1000}\right)+2.7 \times 10^{-16} \exp \left(-\frac{h}{1500}\right)+A \exp \left(-\frac{h}{100}\right)
$$

Where $\mathrm{h}$ is the altitude in meters, $\mathrm{w}$ is the rms wind speed in meters per second, and $\mathrm{A}$ is a nominal value of $\mathrm{C}_{\mathrm{n}}{ }^{2}(0)$ at the ground in $\left[\mathrm{m}^{-2 / 3}\right]$. 
We consider two values of ' $w$ ' that determine the best and worst $\mathrm{C}_{\mathrm{n}}{ }^{2}$ profiles:

- $\quad$ At $\mathrm{w}=10 \mathrm{~m} / \mathrm{s}\left(\mathrm{C}_{\mathrm{n}}{ }^{2}\right.$ best case $)$

- $\quad$ At $w=30 \mathrm{~m} / \mathrm{s}\left(\mathrm{C}_{\mathrm{n}}^{2}\right.$ worst case).

The calculated $\mathrm{C}_{\mathrm{n}}{ }^{2}$ profile is in compliance with measured data [6]. The altitude of the tropopause remains at $13 \mathrm{~km}$ and the maximum altitude of stratosphere is at $26 \mathrm{~km}$. We are interested in the altitude range from 16 to $22 \mathrm{~km}$ where we see one order of magnitude difference between the best case and worst case $\mathrm{C}_{n}^{2}$ profiles as shown in Figure 2. The parabolic $\mathrm{C}_{\mathrm{n}}{ }^{2}$ profile along the link path is shown in Figure 3. The maximum value of $\mathrm{C}_{\mathrm{n}}{ }^{2}$ profile along the Inter-HAP link path is seen at the middle of the link and the average value of $\mathrm{C}_{n}{ }^{2}$ profile increases with the link distance.

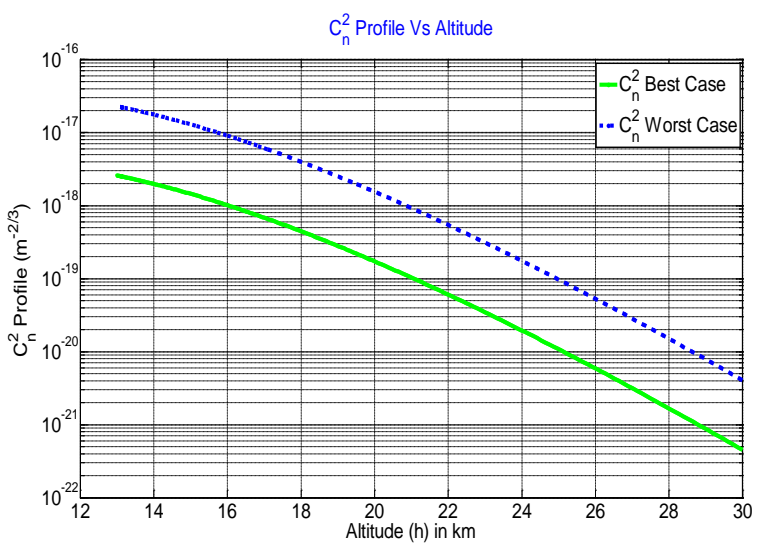

Figure 2. Cn2 profile for best and worst case over altitude using Hufnagel-Valley model

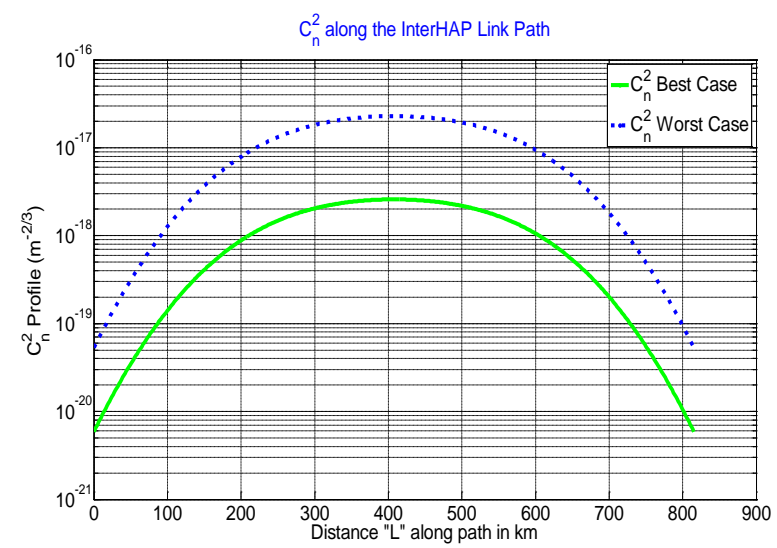

Figure 3. Maximum length Inter-HAP path profile regarding a minimum graze height of $13 \mathrm{~km}$

\subsubsection{Modelling Weak Fluctuation Region}

The performance of an Inter-HAP FSO communication system can be significantly reduced by turbulence-induced scintillation resulting from beam propagation through the atmosphere. Specifically, scintillation can lead to power losses at the receiver and eventually to fading of the received signal below a detectable threshold. The laser beam propagating through the atmosphere will experience intensity fluctuations due to turbulence induced Index of Refraction (IRT) known as scintillation. The intensity scintillation index $\sigma_{\mathrm{I}}^{2}$ is the normalized variance of irradiance fluctuations. The Rytov variance commonly denoted as $\sigma_{\mathrm{R}}{ }^{2}$ is a parameter which defines the areas of fluctuation regimes in the atmosphere. For a variable $\mathrm{C}_{\mathrm{n}}{ }^{2}$ profile, the Rytov variance for a plane wave is defined as [6]:

$$
\sigma_{\mathrm{R}}{ }^{2}=2.25 k^{\frac{7}{6}} \int_{0}^{L} C_{n}^{2}(z)(L-z)^{\frac{5}{6}} d z \text {. }
$$

Where $\mathrm{k}$ is the wave number, which equals $2 \pi / \lambda$, ( $\lambda$ : wavelength), $\mathrm{L}$ is the link distance and $\mathrm{z}$ is the propagation path. According to [1] weak fluctuations are associated with $\sigma_{\mathrm{R}}{ }^{2}<1$, moderate fluctuations with $\sigma_{\mathrm{R}}{ }^{2} \sim 1$ and strong fluctuations with ${\sigma_{R}}^{2}>1$ and the so called saturation regime is defined when ${\sigma_{R}}^{2} \rightarrow \infty$. The Scintillation Index (SI) $\sigma_{I}^{2}$ is defined as 


$$
\sigma_{\mathrm{I}}^{2}=\exp \left[\frac{0.49 \beta_{0}^{2}}{\left(1+0.56 \beta_{0}^{\frac{12}{5}}\right)^{\frac{7}{6}}}+\frac{0.51 \beta_{0}^{2}}{\left(1+0.69 \beta_{0}^{\frac{12}{5}}\right)^{\frac{5}{6}}}\right]-1 .
$$

Where, $\beta_{0}{ }^{2}=0.4 \sigma_{R}{ }^{2}$ for a spherical wave. The SI increases with the increase in $\sigma_{R}{ }^{2}$ initially with the regime of weak fluctuation, which is the region of our scenario defined in the later section 2.2. Basically, weak fluctuation conditions correspond to regimes where the scintillation index $\sigma_{I}^{2}$ throughout the beam profile is less than unity. This Inter-HAP channel model is focused to this condition. The increase in Rytov variance along the beam or link profile with increase in link distance or $\mathrm{C}_{\mathrm{n}}^{2}$ profileis depicted in the Figure 4 for the best and worst case scenarios for a HAP Height (HHAP) of $22 \mathrm{~km}$. We also see the maximum link distances to model the link under weak fluctuation region for a HHAP of $22 \mathrm{~km}$.

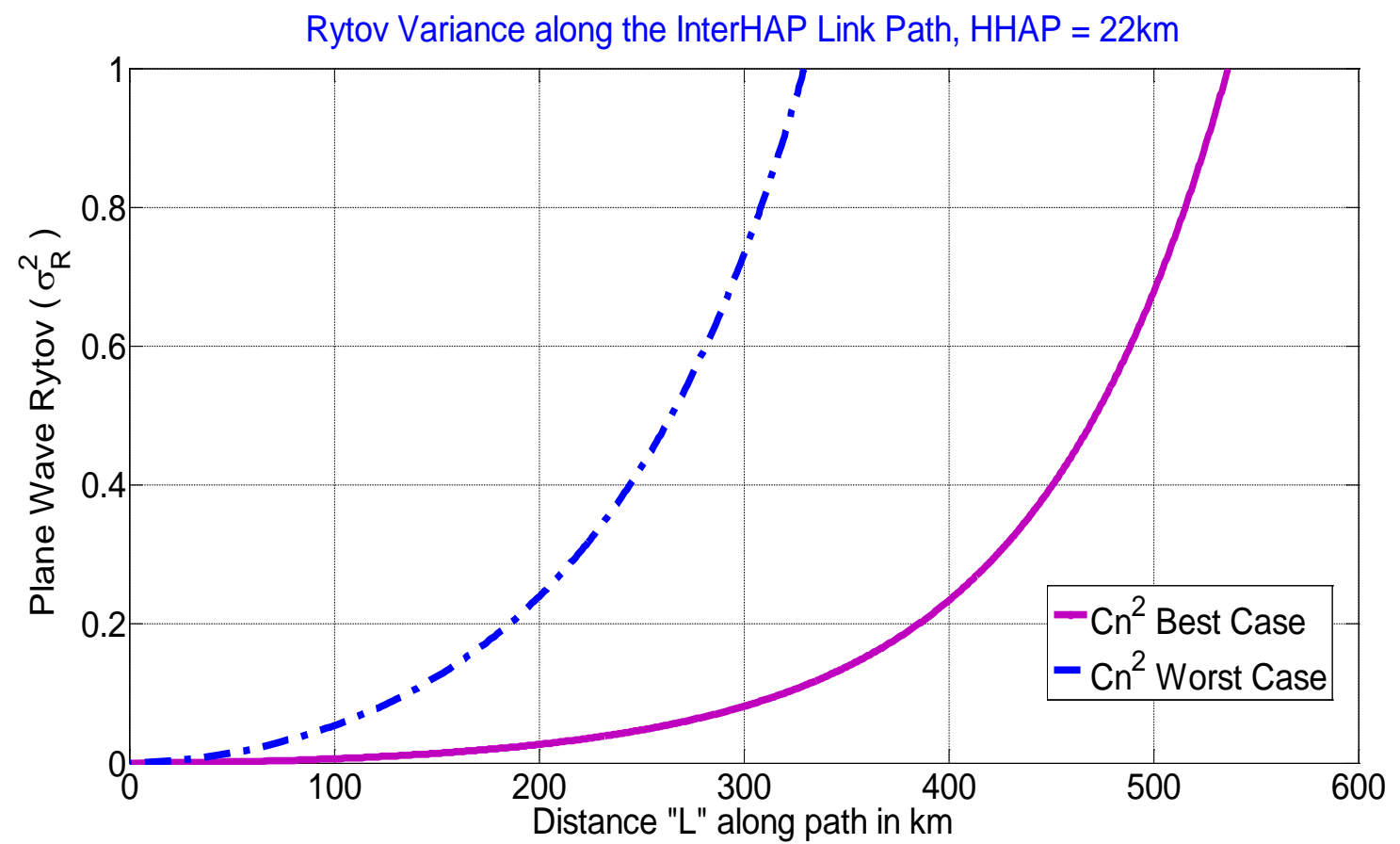

Figure 4. Rytov variance along the Inter-HAP link path

\subsection{Inter-HAP scenario definition and Link Budget}

In this paper, for modelling Inter-HAP channel we define four different scenarios, each positioning two HAPs (the transmitter and the receiver) at an altitude of $22 \mathrm{~km}$. We consider equal HAP Heights (HHAP) and different Graze Heights (GH) where, GH is defined as the minimum height of the FSO link from the earth surface. 
Table 1.Four different Inter-HAP scenarios defined for HAP Heights at $22 \mathrm{~km}$.

\begin{tabular}{|c|c|c|c|c|c|}
\hline Scenario & $\begin{array}{c}\text { Link } \\
\text { Distance } \\
(\mathbf{k m})\end{array}$ & $\begin{array}{c}\text { Graze } \\
\text { Height } \\
\mathbf{( k m )}\end{array}$ & $\begin{array}{c}\text { Rytov } \\
\text { Variance }\end{array}$ & $\begin{array}{c}\text { Scintillation } \\
\text { Index }\end{array}$ & \multirow{2}{*}{$\mathbf{C}_{\mathbf{n}}{ }^{2}$ Case } \\
Short Link - Low SI & 220 & 21.05 & 0.3 & 0.09 & \multirow{2}{*}{$\mathrm{C}_{\mathrm{n}}{ }^{2}$ Worst } \\
Case
\end{tabular}

The third and fourth scenario, defined as Long Link - Low S.I and Long Link - High S.I using $\mathrm{C}_{\mathrm{n}}{ }^{2}$ Best Case, having a link distance of $426 \mathrm{~km}$ and $516 \mathrm{~km}$, is designed to qualify for so called "weak fluctuation" conditions for a minimum and maximum values of Rytov variance less than unity by using a "best case"-turbulence profile defined in section 2.1.1.The first and second scenario, defined as Short Link - Low S.I and Short Link - High S.I uses C $_{n}^{2}$ Worst Case, has a link distance of $220 \mathrm{~km}$ and $339 \mathrm{~km}$, is designed to qualify for so called "weak fluctuation" conditions for a minimum and maximum values of Rytov variance less than unity by using a "best case"-turbulence profile defined in section 2.1.1. The defined scenarios are shown in Table 1 with its corresponding Rytov variance $\sigma_{R}^{2}$ and scintillation index $\sigma_{I}{ }^{2}$ values. From Figure 5, we see four height profiles of the Inter-HAP links for the defined scenarios which shows the placement of both the HAPs (transmitter and receiver) at $22 \mathrm{~km}$ and the variable Graze Height for each link.

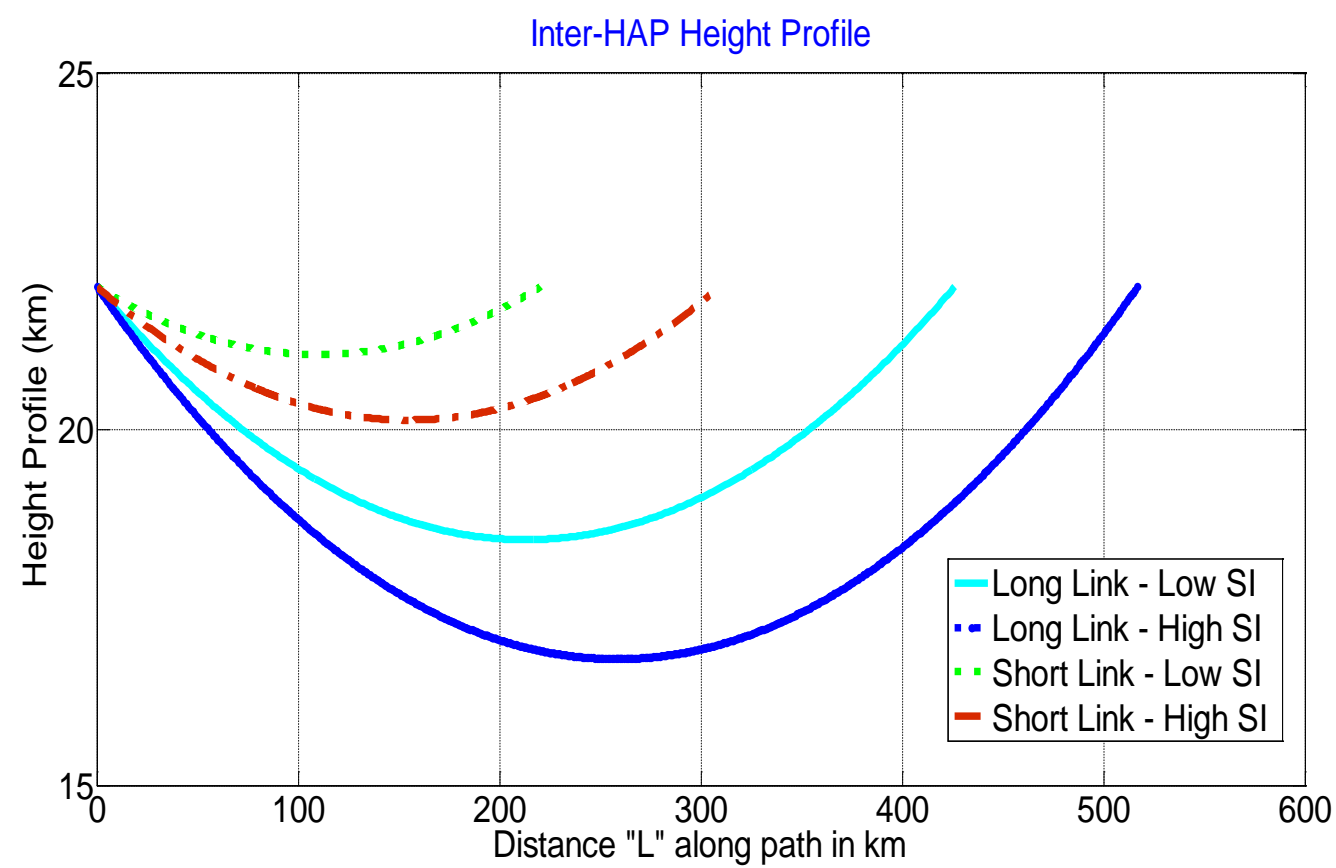

Figure 5. Inter-HAP height profiles for four defined scenarios shows the placement of HAPs (Tx and Rx) at $22 \mathrm{~km}$ and different graze heights.

The link budget calculations for four defined scenarios are shown in Table 2.The mean transmit power is varied to achieve a link margin of $0 \mathrm{~dB}$. 
Table 2. Static Link budget for Inter-HAP link scenario (no scintillation loss regarded):

\begin{tabular}{|c|c|c|c|c|c|}
\hline Parameters & Symbol (Unit) & \multicolumn{4}{|c|}{ Value } \\
\hline Mean Transmit Power & $\mathrm{P}_{\mathrm{Tx}}(\mathrm{mWatt})$ & 44.5 & 119 & 205 & 329 \\
\hline Link Distance & $\mathrm{L}(\mathrm{km})$ & 220 & 339 & 426 & 516 \\
\hline Data Rate & $\mathrm{R}$ (Gbps) & \multicolumn{4}{|c|}{1.25} \\
\hline Wavelength & $\lambda(\mathrm{nm})$ & \multicolumn{4}{|c|}{1550} \\
\hline $\begin{array}{c}\text { Transmitter Divergence, } \\
\text { FWHM }\end{array}$ & $\theta_{\mathrm{Tx}}(\mu \mathrm{rad})$ & \multicolumn{4}{|c|}{85} \\
\hline Rx- Aperture Diameter & $\mathrm{D}_{\mathrm{Rx}}(\mathrm{m})$ & \multicolumn{4}{|c|}{0.15} \\
\hline Atmos. Losses [7] & $\mathrm{a}_{\text {atmos }}(\mathrm{dB})$ & 0.96 & 1.47 & 1.85 & 2.24 \\
\hline $\begin{array}{l}\text { Receiver sensitivity for } \\
\text { BER=1E-9 }\end{array}$ & $\mathrm{S}_{\mathrm{Rx}}(\mathrm{nW})$ & \multicolumn{4}{|c|}{200} \\
\hline Link Margin & $\mathrm{L}_{\mathrm{m}}(\mathrm{dB})$ & \multicolumn{4}{|c|}{0} \\
\hline
\end{tabular}

\subsection{Effects of Pointing Error}

FSO systems require the accuracy of the tracking and pointing capability to be comparable to the divergence angle of the transmitted laser beam. In practice, laser communication terminals operate in the presence of some random angular pointing jitter. So, divergence angle plays an important role for the production of the highest possible average PEP performance [8]. In case of small divergence angles the pointing error is large and the losses due to pointing error reduce as the divergence angle increases. But larger divergence angles lead to higher intensity losses. Therefore, trade-off has to be made between the divergence angle and a reasonable pointing error. Based on [9], we consider pointing error $\sigma_{\text {rms }}=20$ $\mu \mathrm{rad}$ and a full $1 / \mathrm{e}^{2}$ divergence angle of $85 \mu \mathrm{rad}$.

\subsection{Error Probability}

To propose an error correction technique, the Packet Error Probability (PEP) statistics for the modelled Inter-HAP channel are required. At first the Bit Error Probability (BEP) has to be calculated. Based on the fading behavior of the channel the received optical signal has strong variations in its power over time - usually denoted as "Fading". The length of the fades can range from few milliseconds to even hundreds of milliseconds. These fading events increase the BEP because of the reduced power at the receiver. In case of an FSO channel, the bit errors are unevenly distributed over the transmitted data due to the fading and the quantity of BEP depends on the current state of the channel. We have modelled the sources of fades based on IRT effects in the atmosphere and errors due to the presence of pointing error. We consider transmission data rate of $1.25 \mathrm{Gbps}$ and a total packet length of 1000 bits. Also we consider the mean received power is constant for all the scenarios. Figure 6 depicts the calculated BEP with pointing error, with and without fading for the mean received power for two different scintillation index values from Table 1. From the BEP we calculate the PEP which is plotted versus the mean received power as shown in Figure 7. 
Bit Error Probability Vs Rx-Power

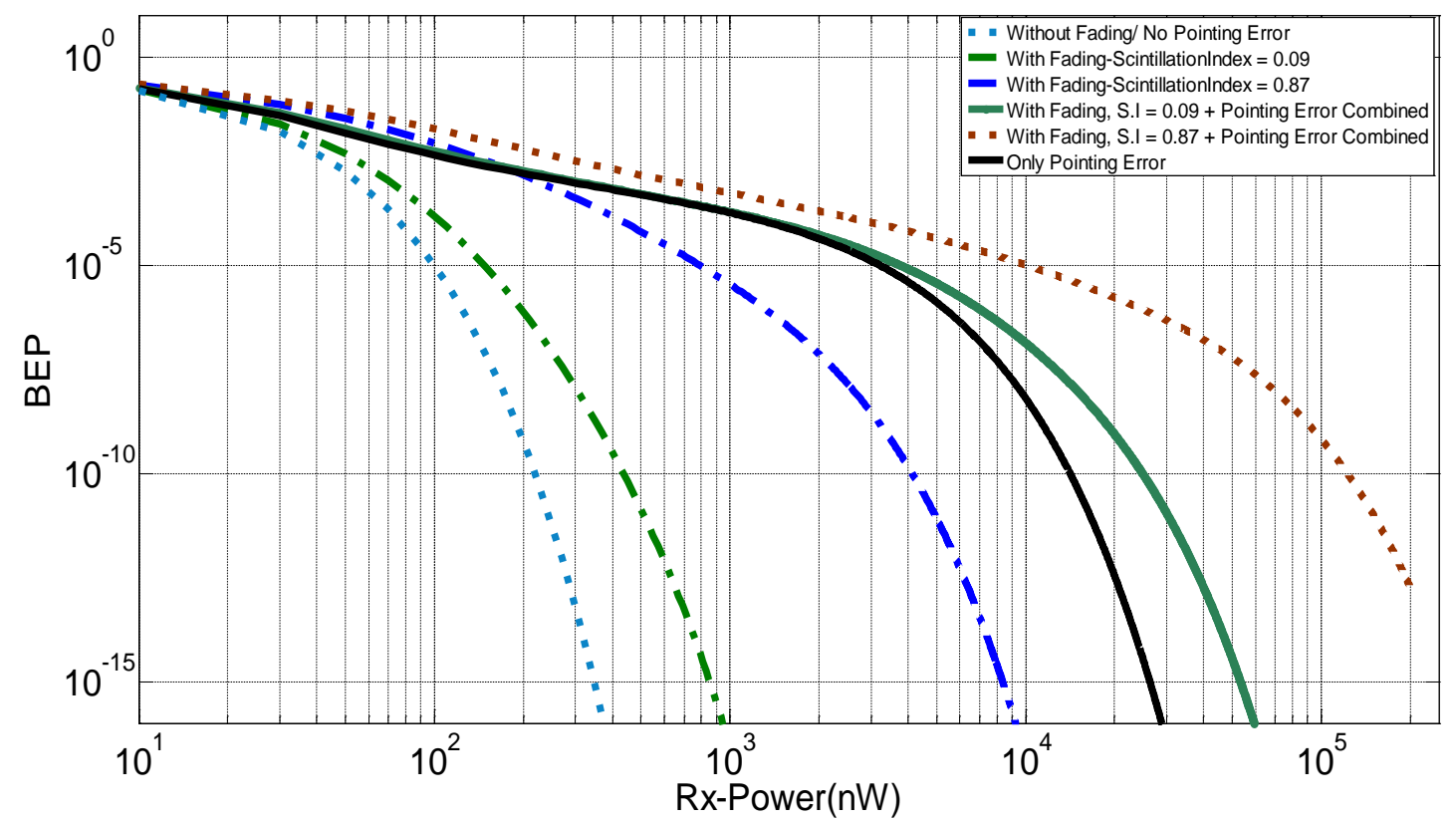

Figure 6. Bit Error Probability (BEP) for different mean Rx-power in (nW)

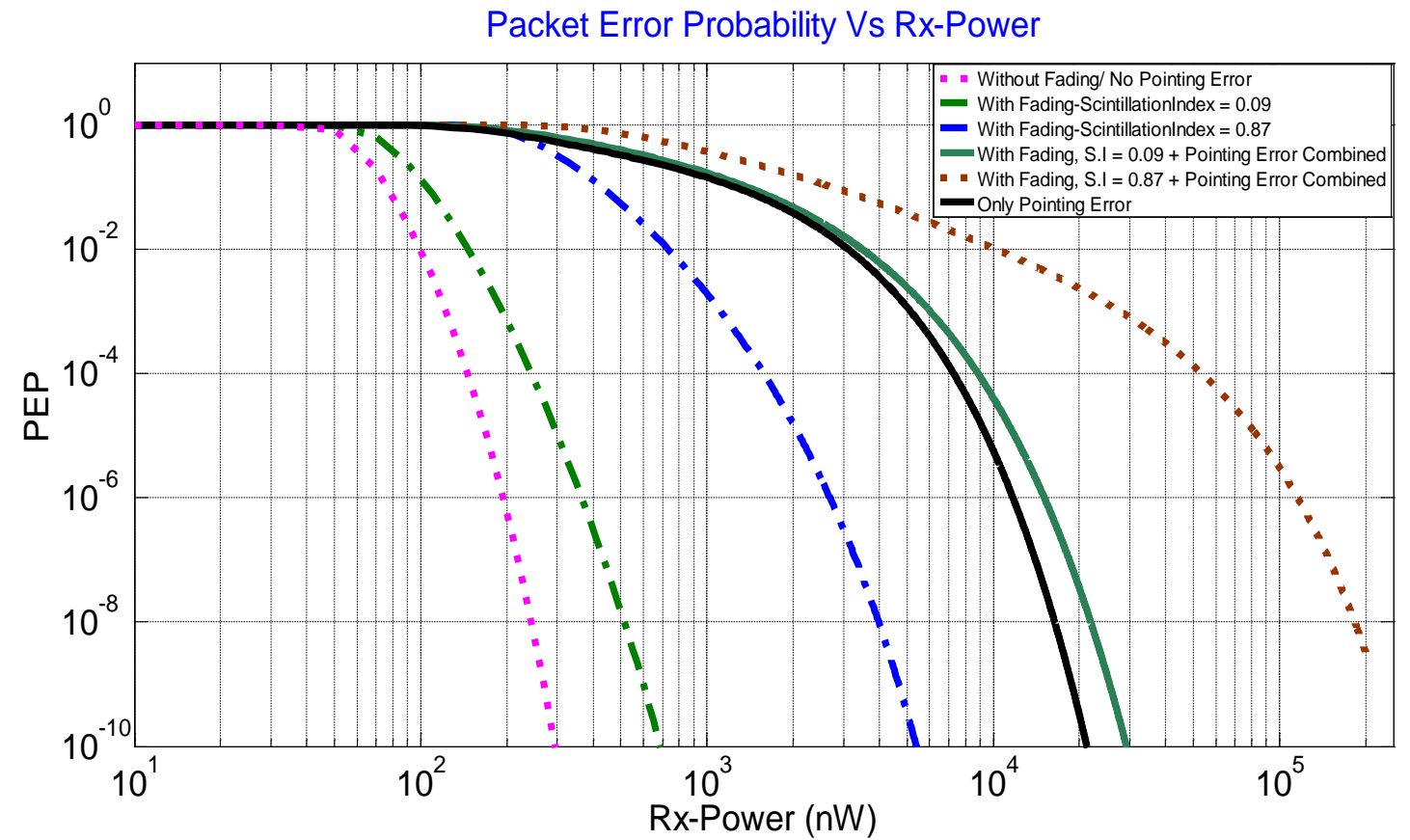

Figure 7. Packet Error Probability (BEP) for different mean Rx-power in (nW).

We are interested in the PEP range from $10^{-6}$ to $10^{-2}$ and the future sections will care only for this range of PEP when calculating the performance characteristics of different ARQ schemes. 


\section{ARQ SCHEMES}

Error detection and error correction are the basic blocks of error control mechanisms for communication systems. Error control in the data link layer is based on protocols denoted as Automatic Repeat reQuest (ARQ) [10]: The destination requests the source to retransmit a packet when it is incorrectly received or missing. In case of an Inter-HAP bidirectional channel, the backchannel can be used to inform the partner HAP.

\subsection{Selected ARQ Schemes}

In general, ARQ schemes can be classified [11] into Stop-and-Wait ARQ (SW-ARQ) and window-based ARQ with socalled Go-back-N (GBN-ARQ) or Selective Repeat (SR-ARQ) retransmission strategies.

SW-ARQ is the simplest type of error control mechanism. The transmitter sends a packet to the receiver and waits until the Acknowledgement packet (ACK) arrives from the receiver. In case of a missing ACK, a timeout triggers the retransmission of the packet to the receiver. This type of ARQ is the simplest and easiest to implement but inefficient since the transmitter spends a lot of time waiting for each packet to be acknowledged by the receiver. This inefficiency can be mitigated by windowing (pipelining) where multiple packets are allowed to be emitted during the time that the transmitter waits for an ACK. The transmitter continues the sending of packets without waiting for ACKs up to the window size. When the window size is reached the transmitter waits for ACKs for the transmitted packets. As soon as the packet at the lower end of the window gets ACKed, the window is advanced and a new packet can be sent. Also with windowing the reception of ACKs is controlled by timer(s). In the case of GBN-ARQ, at any single missing (i.e., timerout), ACK the transmitter re-transmits the complete unacknowledged window to the receiver. In case of noisy channels and channels with large bandwidth-delay products, GBN-ARQ is not suitable since the whole window of packets has to be re-transmitted for any lost or damaged packet or ACK using up much of the channel capacity and thereby reducing transmission efficiency. SR-ARQ overcomes the inefficiency of GBN-ARQ by re-transmitting only those packets in the window which were not ACKed. However, SR-ARQ is also very complex to implement due to its non-sequential storage management at the receiver.

\subsection{ARQ Parameter Definition}

In this paper, we define the values of ARQ parameters required to calculate the transmission efficiency (defined as the fraction of the channel bit rate being usable for payload transport) of each ARQ scheme as shown in Table 3. The parameters defined are $\mathrm{N}_{\text {packet }}$ the total length of a packet transmitted, $\mathrm{N}_{\mathrm{o}}$ the number of overhead bits in a packet, $\mathrm{N}_{\mathrm{a}}$ the number of ACK bits, $\mathrm{N}_{\text {info }}$ the number of information or payload bits in a packet, $\mathrm{R}$ the bit rate of the channel, $\mathrm{L}$ the link distance, $t_{\text {prop }}$ the propagation time and $t_{\text {proc }}$ is the processing time of the system to prepare the ACK frame which is assumed as 0 neglecting the processing time at the destination.

Table 3. ARQ parameters.

\begin{tabular}{|c|c|}
\hline Parameters & Value \\
\hline $\mathrm{N}_{\text {packet }}$ & 1000 bits \\
\hline $\mathrm{N}_{\mathrm{o}}$ & 80 bits \\
\hline $\mathrm{N}_{\mathrm{a}}$ & 16 bits \\
\hline $\mathrm{N}_{\text {info }}$ & $920 \mathrm{bits}$ \\
\hline $\mathrm{R}$ & $1 \mathrm{Gbps}$ \\
\hline $\mathrm{L}$ & $600 \mathrm{~km}$ \\
\hline $\mathrm{t}_{\text {prop }}$ & $0.6 \mathrm{~ms}, 1 \mathrm{~ms}, 2 \mathrm{~ms}$ \\
\hline $\mathrm{t}_{\text {proc }}$ & 0 \\
\hline
\end{tabular}




\subsection{Transmission Efficiency of Selected ARQ Schemes}

This section discusses the performance differences of the selected ARQ schemes based on their transmission efficiency presenting a quantitative comparison. From Section 2, the Inter-HAP FSO channel is modelled as a bi-directional symmetrical channel. This results in the same PEP on both the forward and backward channel. For calculations here, we consider the information transmission is unidirectional and the back channel is used only for ACK. Also, we use the value of parameters in Table 2. The vital factors determining the performance of the system are Packet Length $\left(\mathrm{N}_{\text {packet }}\right.$ ), Bandwidth Delay Product (BDP) and Packet Error Probability (PEP) [9]. The packet length and PEP are known from the Section 3.2 and Section 2.4 respectively. BDP is the product of the data rate of the channel and the twice of the sum of propagation delay $t_{\text {prop }}$ and the processing time $t_{\text {proc }}$. This includes both the forward and the backward channel. The transmission efficiency of the three selected ARQ schemes is depicted in Figure 8. The performance of SW-ARQ was calculated for a link distance of $600 \mathrm{~km}$ and for comparison for different date rates of $100 \mathrm{Kbps}, 1.5 \mathrm{Mbps}, 50 \mathrm{Mbps}$ and 1Gbps. SW-ARQ shows very low performance for high BDP: Its transmission efficiency fails at a link distance of 600 $\mathrm{km}$ for data rates of 1 Gbps and even 50 Mbps since: each individual packet has to be ACKed successfully before the next can be sent out. In case of GBN-ARQ in our scenario, the transmission efficiency decreases for larger window sizes. For the interesting range of PEP from $10^{-6}$ to $10^{-2}$, rather small window sizes would have to be selected - also leading to an under-utilized channel. SR-ARQ shows the best performance in the interesting range of PEP and deteriorates as PEP becomes larger than $10^{-2}$.
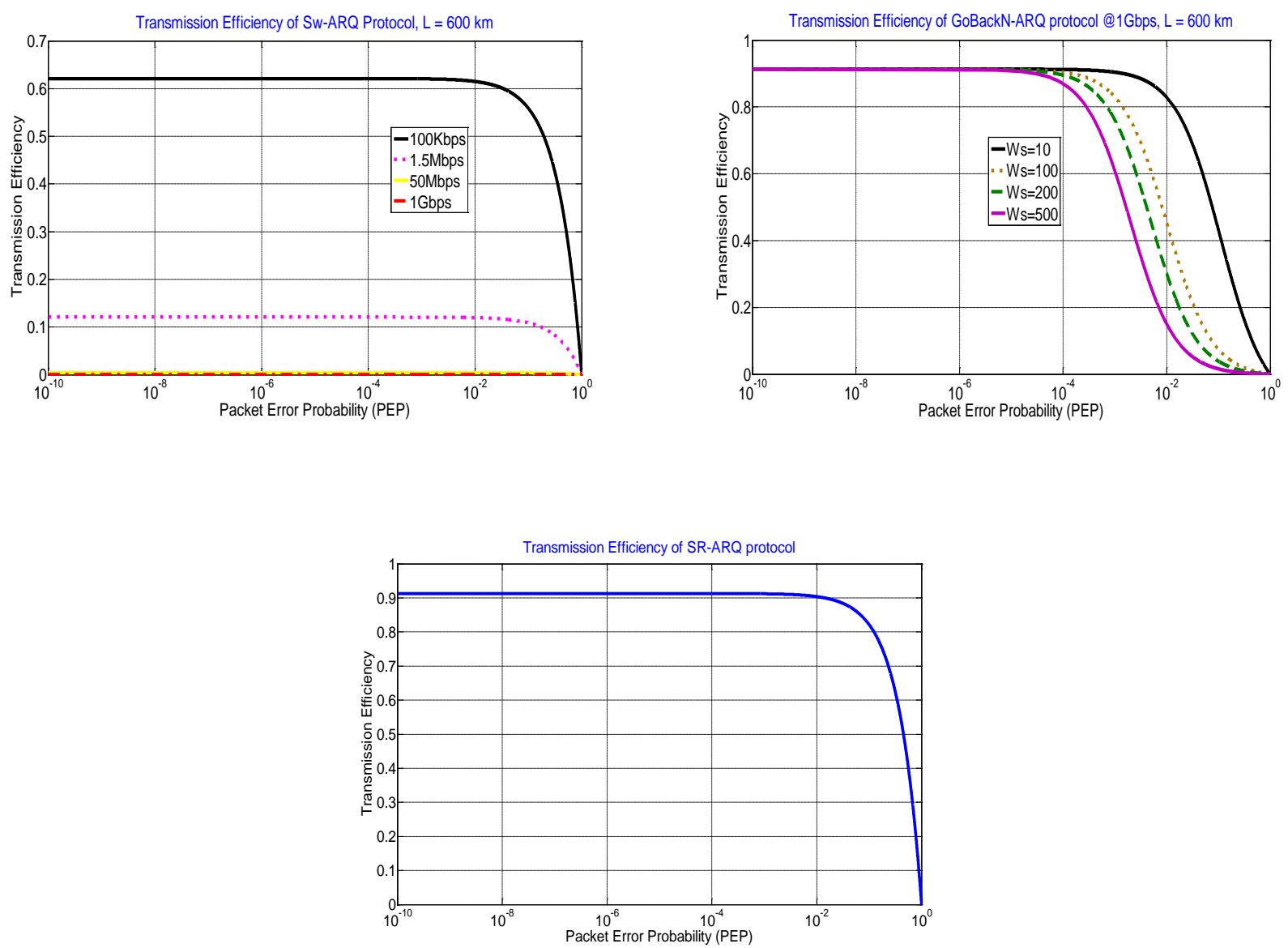

Figure 8. Transmission Efficiency of ARQ schemes. 


\section{RESULTS}

In this section, using the previously described Inter-HAP scenario, we analyze the performance of three selected ARQ schemes. Figure 9 compares the results in terms of transmission efficiency for Inter-HAP Long Link - High S.I scenario. The purpose is to find the maximum transmission efficiency achievable under the impact of atmospheric turbulence and pointing error characterized in terms of PEP. More specifically, Figure 9 shows the Packet Error Probability (PEP) versus the transmission efficiency for a link distance $\mathrm{L}=516 \mathrm{~km}$, Data rate $\mathrm{R}=1 \mathrm{Gbps}$, Scintillation Index (S.I) $\sigma_{\mathrm{I}}^{2}=$ 0.87 , including pointing error $\sigma_{\mathrm{rms}}=20 \mu \mathrm{rad}$ for different selected ARQ schemes. It is clearly visible that the performance of SW-ARQ fails in this scenario. In case of GBN-ARQ, the maximum performance is visible until PEP of $10^{-5}$ and then starts to degrade as the PEP increases reaching to the lowest for approximately at $10^{-2}$. In case of SR-ARQ, the maximum performance is achieved until PEP of $10^{-2}$. The performance again degrades as the PEP increases. This allows us to select the suitable ARQ scheme for the given channel based on its PEP.

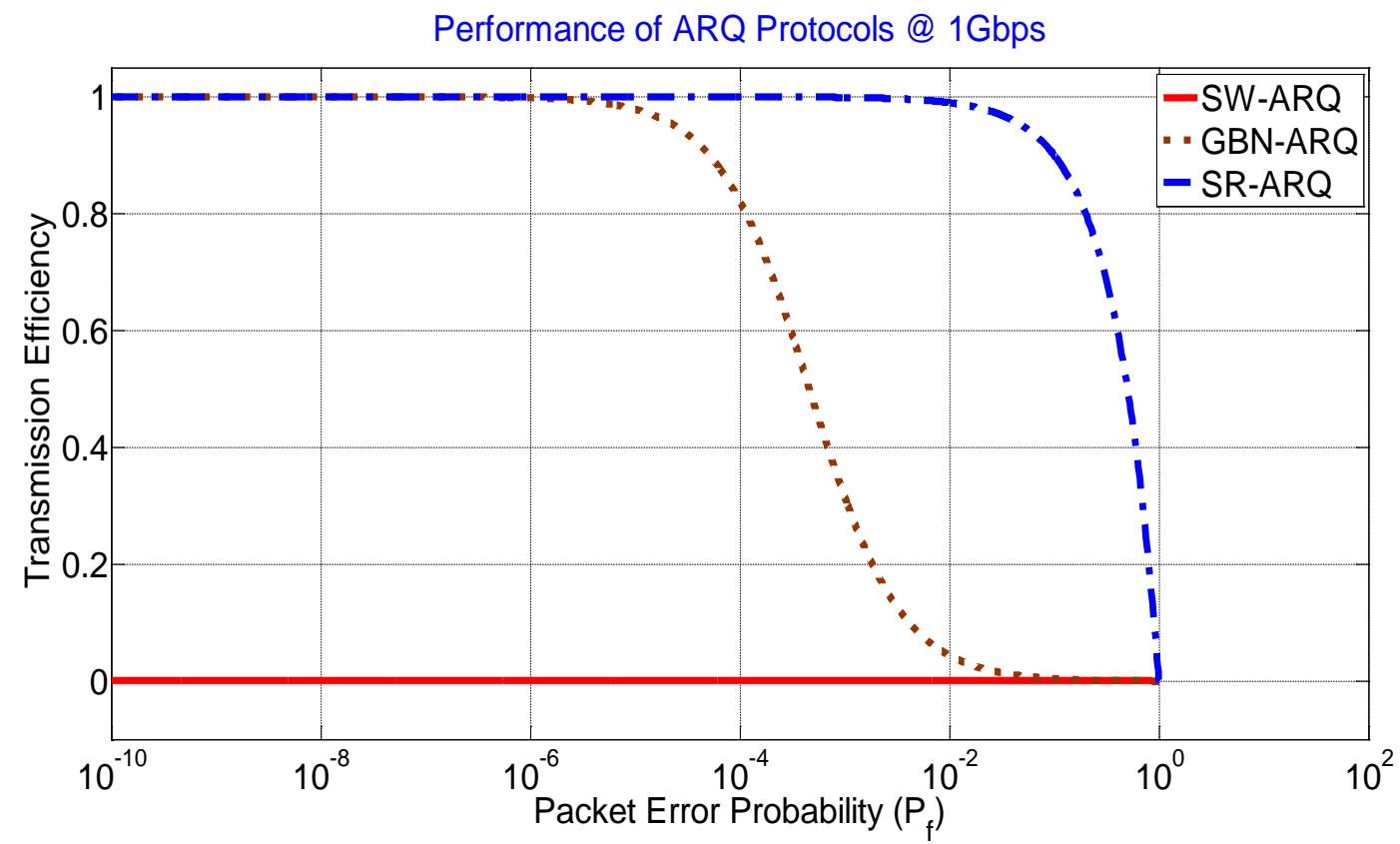

Figure 9.Transmission Efficiency comparison of selected ARQ schemes - Long Link - High SI scenario

Table 4.Selected ARQ schemes for maximum transmission efficiency depending on PEP for different modeled Inter-HAP scenarios.

\begin{tabular}{|c|c|c|c|c|}
\hline Scenario & $\begin{array}{c}\text { Link } \\
\text { Distance } \\
\mathbf{( k m )}\end{array}$ & SW-ARQ & GBN-ARQ & SR-ARQ \\
\hline Short Link - Low SI & 220 & $\mathrm{x}$ & $10^{-5}$ & $10^{-2}$ \\
\hline Short Link - High SI & 339 & $\mathrm{x}$ & $10^{-5}$ & $10^{-2}$ \\
\hline Long Link - Low SI & 426 & $\mathrm{x}$ & $10^{-5}$ & $10^{-2}$ \\
\hline Long Link - High SI & 516 & $\mathrm{x}$ & $10^{-5}$ & $10^{-2}$ \\
\hline
\end{tabular}

From Table 4, we see that both GBN-ARQ and SR-ARQ show the best fit to our model because of its higher transmission efficiency for PEP until $10^{-5}$ and $10^{-2}$, whereas failed performance of SW-ARQ cannot be considered. 


\section{DISCUSSION AND CONCLUSION}

In this work, a bi-directional symmetrical Inter-HAP FSO link has been modeled for different scenarios. These modeled scenarios were simulated in terms of optical scintillation induced due to IRT and in presence of pointing error. Based on the packet error probability (PEP) we have analytically studied the performance of selected ARQ schemes for Inter-HAP link scenarios. Numerical results showed that that IRT effects, pointing error, and link distance affect the transmission efficiency of the ARQ schemes. The fading behavior of the link and identical error-rate of both forward and backward channel can lead to a precise design of ARQ scheme. Thus, for the investigated Inter-HAP scenarios the usage of GBNARQ and SR-ARQ schemes with appropriate window sizes and packet sizes could significantly increase the transmission efficiency. The presented approach can be improved taking also the channel reciprocity of the Inter-HAP link into consideration meaning the received signal levels at both link ends are similar or, ideally identical [12]. This approach will also be further extended to choose an appropriate Forward Error Correction (FEC) scheme as a part of error correction technique. This leads to an option of designing a hybrid error detection and correction system.

\section{REFERENCES}

[1] Andrews, L.C and Phillips, R.C., [Laser Beam Propagation through Random Media - Second Edition], SPIEPress, Bellingham, (2005).

[2] Tozer, T. C., Grace, D., "High-altitude platforms for wireless communications," Electron. Commun. Eng. J., 13, 127-137 (2001).

[3] Fidler, F., Knapek, M., Horwath, J., Leeb, W., "Optical Communications for High-Altitude Platforms," Selected Topics in Quantum Electronics IEEE Journal of, 16, 1058-1070 (2010).

[4] Giggenbach, D., Purvinskis, R., Werner, M., Holzbock, M., "Stratospheric Optical Inter-Platform Links for High Altitude Platforms," Online Proceedings. 20th AIAA International Communications Satellite Systems Conference, (2002).

[5] Andrews, L.C and Phillips, R.C., [Laser Beam Propagation through Random Media - Second Edition], SPIEPress, Bellingham, 480-481 (2005).

[6] Beland, R., "Propagation through atmospheric optical turbulence," in IR/EO Handbook, Vol 2, SPIE Press Bellingham, Washington, 157-232 (1993).

[7] Giggenbach, D., "Optimierung der optischen Freiraumkommunikation durch die turbulente Atmosphäre - Focal Array Receiver," Univ. der Bundeswehr, Diss., 67-68 (2004).

[8] Toyoshima, M., Jono, T., Nakagawa, K., and Yamamoto, A., "Optimum divergence angle of a Gaussian beam wave in the presence of random jitter in free-space laser communication systems," J. Opt. Soc. Am. A 19, 567571 (2002).

[9] David, F., Giggenbach, D.,Henniger, H.,Horwath, J.,Landrock, R.,Perlot, N., "Design Considerations for Optical Inter-HAP Links," Proceedings ICSSC, 22nd AIAA International Communications Satellite Systems Conference \& Exhibit, Monterey, CA, May 9-12, (2004).

[10] Leon-Garcia, A., Widjaja, A., [Communication Networks: Fundamental Concepts and Key Architectures], New York, NY, USA: McGraw-Hill, Inc., (2003).

[11] Shu Lin, D. J. C., [Error Control Coding: Fundamentals and Applications -Second Edition], Hemel Hempstead, Herts, UK: Prentice-Hall International, (1983).

[12] Giggenbach, D., Cowley, W., Grant, K., Perlot, N., "Experimental verification of the limits of optical channel intensity reciprocity". Applied Optics, 51 (16), OSA - Optical Society of America, 3145-3152 (2012). 